\title{
Probing Sagittarius A* accretion with ALMA
}

\author{
Elena Murchikova \\ TAPIR, California Institute of Technology, \\ MC 350-17, Pasadena, CA 91125, USA \\ email: lena@caltech.edu
}

\begin{abstract}
The submm Hydrogen recombination line technique can be used as a probe of the Galactic Center. We present the results of our $\mathrm{H} 30 \alpha$ observations of ionized gas from within $0.015 \mathrm{pc}$ around SgrA*. The observations were obtained on ALMA in cycle 3. The line was not detected, but we were able to set a limit on the mass of the cool gas $\left(T \sim 10^{4} \mathrm{~K}\right)$ at $2 \times 10^{-3} M_{\odot}$. This is the unique probe of gas cooler than $T \sim 10^{6} \mathrm{~K}$ traced by X-ray emission. The total amount of gas near SgrA* gives us clues to understanding the accretion rate of SgrA*.
\end{abstract}

Keywords. Galaxy: center, ISM: lines and bands, black hole physics.

\section{Introduction}

Our galactic center black hole, Sagittarius A* (SgrA*) [Ghez et al. (2000)], is quiet and predominantly uneventful, but the closet to us supermassive black hole. It is therefore our best hope to study accretion, black hole feedback and, potentially, physics in strong gravitation fields.

The galactic center is a very complex region. Huge dust extinction prevents us from seeing directly into it at short wavelength. Many important properties of the galactic center are largely unknown. Among them is the accretion rate into SgrA* and the total amount of gas in its accretion zone. Chandra observations in X-ray probe very hot gas at $T \sim 10^{6} \mathrm{~K}$, the amount of which $\sim 10^{-3} M_{\odot}$. The amount of cool gas, in this context defined as not-X-ray-emitting, is unknown.

The goal of our observations is to probe the amount of cool ionized gas at characteristic temperature of $T \sim 10^{4} \mathrm{~K}$ in the immediate proximity of $\mathrm{SgrA}^{*}$ and, using it, estimate the accretion into the black hole. As the probe we use submm recombination line of Hydrogen, which has negligible extinction and bring us direct information about the workings of inner part of the galactic center region. Submm wavelengths are long enough to avoid substantial dust extinction, and short enough to be unaffected by free electron scattering. In this note we find that there are less than $\sim 2$ Jupiter masses of cool gas in the accretion zone around SgrA*. This estimation comes from the limit set by nondetection of Hydrogen recombination line. The observations were obtained with ALMA in cycle 3 (partially completed). We then conduct an order of magnitude calculations and conclude that the amount of accretion on $\mathrm{SgrA}^{*}$ is less than $\sim 10^{-6} M_{\odot} y r^{-1}$.

\section{The basics of the recombination line technique}

Let us first discuss how the recombination line technique works. Assume there is a constant source of ionizing radiation surrounded by neutral Hydrogen. If the source emits photons with energy at or above $13.6 \mathrm{eV}$, it ionizes Hydrogen $H \rightarrow p^{+}+e^{-}$. Free electrons and protons then can recombine. The rate of recombination is proportional to 


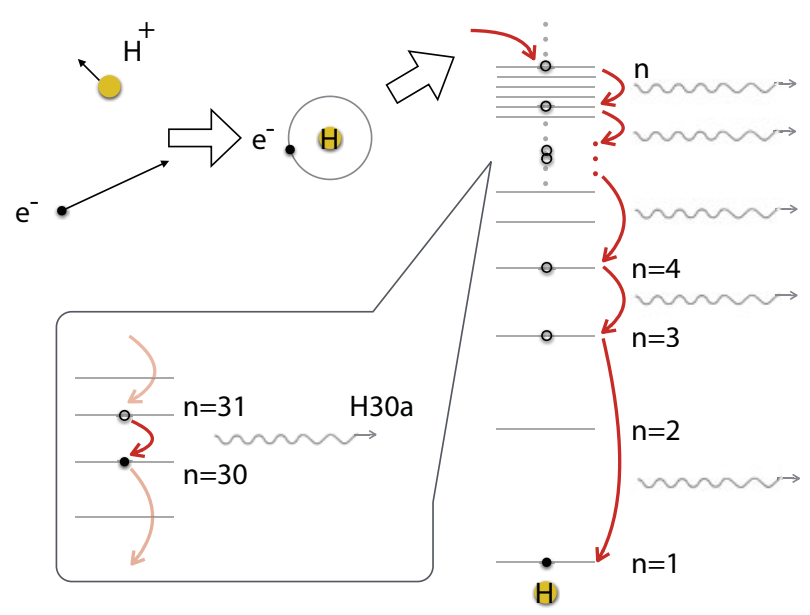

Figure 1. The recombination line technique illustration.

the density squared of the particles and the frequency of the encounters, determined by their relative velocity and interaction cross-section. In equilibrium the ionization rate will be equal to the recombination rate and to the number of ionizing photons emitted per unit time: $n_{\text {ionization }}=n_{\text {recomb }}=n_{\gamma}$.

Some recombinations happen into one of high principal quantum number $n$ level. Electron then quickly cascades down to the bottom via series of random (permitted) transitions. Each transition in the cascade is accompanied by the emission of a photon with energy equivalent to the energy difference between the levels. See Figure 1 for illustration of recombination process. Among all the electrons cascading, some will pass through a pair or level we are interested in. In this paper it is $H 30 \alpha-$ the transition from Hydrogen level $n=31$ to level $n=30$. (We discuss the reasons for selecting $H 30 \alpha$ below.)

The electron capture and each transition in cascade are completely described by quantum mechanics. Arriving at the telescope $H 30 \alpha$ photons carry direct information of how many recombination cascades are happening, how many atom are recombining and, assuming reasonable assumptions of gas density and temperature, how many ionized atoms are in the region.

Detail discussion of the recombination processes, cascades and transition probabilities can be found in Scoville \& Murchikova (2013). Below we present a summary of the relations important for out present discussion. The velocity integrated line flux $(S \Delta V)$ of a given transition can be expressed as

$$
S \Delta V=\frac{\epsilon_{\text {line }} \mathrm{EM}}{4 \pi D^{2}} \frac{c}{\nu_{\mathrm{obs}}} 10^{18} \mathrm{Jy} \mathrm{km} \mathrm{sec}^{-1} .
$$

Here $\epsilon_{\text {line }}$ is the line emissivity, $E M=n_{e} n_{\text {ion }} \times$ Volume $_{\mathrm{HII}}$ is the emission measure defined, $\mathrm{D}$ is the distance to the source, $\nu_{\mathrm{obs}}$ observing frequency, $c$ is the speed of light, and the factor of $10^{18}$ is the scaling between CGS units and $\mathrm{Jy} \mathrm{km} \mathrm{sec}{ }^{-1}$. The distance to $\mathrm{SgrA}^{*}$ is $D=7.9 \mathrm{kpc}$, the frequency of $H 30 \alpha$ transition is $\nu_{\mathrm{obs}}=231.9 \mathrm{GHz}$, and taking line emissivity at characteristic temperature $T=10^{4} \mathrm{~K} \epsilon_{H 30 \alpha}=1.05 \times 10^{-31} \mathrm{erg} \mathrm{sec}^{-1}$ we get

$$
S \Delta V_{H 30 \alpha}=1.8 \times 10^{-60} \mathrm{EM} \mathrm{Jy} \mathrm{km} \mathrm{sec}^{-1} .
$$

The emission measure is the product of the electron and ion densities integrated over volume of ionized gas. It can be re-expressed in terms of the gas mass and its density, 
assuming $n_{e} \simeq n_{p}$ and using $M_{\mathrm{HII}}=m_{p} n_{p} \times$ Volume $_{\mathrm{HII}}$

$$
\mathrm{EM}=1.2 \times 10^{60} \frac{M_{\mathrm{HII}}}{10^{-3} M_{\odot}} \frac{n_{p}}{10^{6} \mathrm{~cm}^{-3}} .
$$

Substituting this into the expression for the velocity integrated line flux, we find

$$
S \Delta V_{H 30 \alpha}=2.2 \frac{M_{\mathrm{HII}}}{10^{-3} M_{\odot}} \frac{n_{p}}{10^{6} \mathrm{~cm}^{-3}} \mathrm{Jy} \mathrm{km} \mathrm{sec}^{-1} .
$$

This is a useful relation between the velocity integrated line flux and the mass of HII near the ionizing source, which we are going to use in the following section.

The approach discussed in this section is applicable to any recombination line without maser amplification. There is, however, an advantage of using Hydrogen recombination line. Most of the material in the Universe is Hydrogen, while the abundances of other elements in a given region are largely unknown. Choosing Hydrogen recombination line saves us from dealing with metallicity uncertainties. The advantage of choosing submm line was discussed in the introduction. Particular choice of $H 30 \alpha$ dictated by its relative clearance from strong molecular emission and absorption lines.

\section{The observations}

The data for this project was obtained on ALMA, project 2015.1.00311.S. The data was taken on April 15, 2016 in C36-2/3 configuration with 43 12m antennas. Min-max baseline: $15 \mathrm{~m}-460 \mathrm{~m}$. Beam size is 0.8 arcsec. Maximum recoverable scale is 24.7 arcsec. The project was half observed with integration on target $37 \mathrm{~min}$. Half power beam size corresponds to $0.03 \mathrm{pc}$ in the galactic center. The calibration and data reduction was performed at NAASC. The provided script was used by the author to perform recalibration and re-reduction with more accurate flagging. The data were imaged using CASA. The line was not detected, therefore only the limit was set.

\section{Amount of the material and the limit on the accretion rate}

The line was not detected in the observations, but we were able to set the limit on the peak flux at $S_{H 30 \alpha} \leqslant 3 \mathrm{mJy}$. Assuming now that the line width should be of order $\Delta V \sim 1000 \mathrm{~km} \mathrm{sec}^{-1}$ and using expression 2.4, we conclude that the mass of the gas withing $0.015 \mathrm{pc}$ (the radius of the beam) around $\mathrm{SgrA}^{*}$ is

$$
M_{<0.015 \mathrm{pc}} \leqslant 2 \times 10^{-4} M_{\odot}=2 M_{\text {Jupiter }} .
$$

Note once again, that in order to obtain this number we made the assumption that characteristic temperature of the gas is $\sim 10^{4} \mathrm{~K}$ and the characteristic density of the ionized gas is $10^{6} \mathrm{~cm}^{-3}$.

Let us now put this number into perspective of accretion rate on SgrA*. The accretion averaged over the lifetime of our galaxy, which can be obtained by dividing the $3.7 \times$ $10^{6} M_{\odot}$ mass of SgrA* [Schödel, Merritt \& Eckart (2009)] by the age of the galaxy is $\sim 10^{-4} M_{\odot} \mathrm{yr}^{-1}$. Assuming that it takes the gas $\sim 1000$ years to fall from $0.015 \mathrm{pc}$ we end up with the mass

$$
M_{<0.015 \mathrm{pc}}^{\text {averaged accretion }} \leqslant 0.5 M_{\odot}=500 M_{\mathrm{Jupiter}}
$$

within 0.015 pc from SgrA*, which is much greater than our limit and can be ruled out.

The current accretion rate [Melia \& Falcke (2001) and Genzel et al. (2010)] ranges 
between $10^{-9}$ and $10^{-6} M_{\odot} \mathrm{yr}^{-1}$. Using the upper limit we get

$$
M_{<0.015 \mathrm{pc}}^{10^{-6} \mathrm{M}} \mathrm{yr}^{-1} \leqslant 5 \times 10^{-3} M_{\odot}=5 M_{\text {Jupiter }}
$$

withing $0.015 \mathrm{pc}$, which is the limiting number we get from out observations.

With more data to come in cycle 4 we are hopeful that we achieve considerable improvement over this limit.

\section{Conclusion and Future Prospects}

The total amount of gas near SgrA* is widely unconstrained, at the moment. Knowing the total amount of gas near the black hole is important to understanding its accretion rate. We attempted provide a direct insight into this issue, by observing submm recombination line of Hydrogen $H 30 \alpha$ from 0.015 pc radius around it. The line was not detected, but we we able to set the $<2 \times 10^{-3} M_{\odot}=2 M_{\text {Jupiter }}$ limit on the amount of cool non-X-ray-emitting gas in the region. This limit provide order of magnitude quality constraint on $\mathrm{SgrA}^{*}$ accretion at $\dot{M} \sim 3 \times 10^{-7} M_{\odot}$, assuming $T \sim 10^{4} \mathrm{~K}$ and density $10^{6} \mathrm{~cm}^{-3}$.

In order to obtain quantitative estimations we have to assume characteristic temperature and density of the gas. Such approach unavoidably carry uncertainties. This happens because the velocity integrated line flux is a complicated function of temperature and density. Namely, density explicitly enters EM, see equation 2.3, and temperature dependence is hidden in the emissivity $\epsilon_{\text {line }}$ (for Hydrogen recombination lines $n<50$ in the proximity of $T=10^{4} \mathrm{~K}$, it scales as $\epsilon \sim T^{-4 / 3}$ Scoville \& Murchikova (2013)). Density dependence of emissivity is negligible. There is however a way of minimizing uncertainties from assuming characteristic temperature and density of the gas. We can use data from simulations, for example, Russell, Wang, \& Cuadra (2016), which does careful account of the gas behavior in the immediate proximity of $\mathrm{SgrA}^{*}$ and record information about the density and the temperature of the gas. Implementation of this approach is in progress.

This proceeding shall be treated as a demonstration of concept rather than a stand alone result report.

\section{Acknowledgments}

I am grateful to Nick Scoville, Jin Koda, Yuri Levin and Sterl Phinney for discussion and to Dr. David and Barbara Groce for their kindness and support. This paper makes use of the following ALMA data: ADS/JAO.ALMA\# 2015.1.00311.S. ALMA is a partnership of ESO (representing its member states), NSF (USA) and NINS (Japan), together with NRC (Canada) and NSC and ASIAA (Taiwan), in cooperation with the Republic of Chile. The Joint ALMA Observatory is operated by ESO, AUI/NRAO and NAOJ

\section{References}

Scoville, N. \& Murchikova, E. 2013, ApJ, 779, 75

Ghez, A. et al. 2000, Nature, 407, 349

Schödel, R., Merritt, D., \& Eckart, A. 2009, A\& A, 502, 91

Genzel, R. et al. 2010, Rev Mod Phys, 82, 3121

Melia, F. \& Falcke, H. 2001, ARAA, 39, 309

Russell, C. M. P., Wang, Q. D., \& Cuadra, J. 2016, arXiv:1607.01562 